\title{
A new approach to obtain a snapshot of human gut microbial proteolysis signatures
}

Zhixiang Yan

Feixiang He

Fei Xiao

Huanhuan He

Dan Li

Li Cong

Lu Lin

Huijin Zhu

Yanyan Wu

Ru Yan

Xiaofeng Li

Hong Shan

\section{Video Byte}

Keywords: Metaproteomics, Gut microbial proteolysis, Inflammatory bowel disease, IBD, gut microbes, Microbiome

Posted Date: February 25th, 2021

DOI: https://doi.org/10.21203/rs.3.rs-276046/v1

License: (9) This work is licensed under a Creative Commons Attribution 4.0 International License. Read Full License 


\section{Abstract}

Our gut microbes must respond rapidly to dynamic intestinal environments. Rapidly degrading misfolded proteins through proteolysis and activating regulatory proteins helps them to maintain homeostasis. However, alterations in microbial proteolytic function have not been investigated in complex disease states like inflammatory bowel disease (IBD). A recent study identified a new technique to obtain snapshots of human gut microbial proteolysis under various conditions. The technique, called semitryptic peptide centric metaproteomic mining, uses a comprehensive meta-database and high-resolution datasets. Using two published large-scale datasets, researchers were able to obtain potential signatures of altered gut microbial proteolysis in patients with IBD. Functional alterations were primarily in microbial carbohydrate transport and metabolism, oxidative stress, cell motility, protein synthesis, and maturation. IBD-affected proteolysis signatures were concentrated in the terminal ileum for Crohn's disease and in the descending colon for ulcerative colitis and protease inhibitors and immunoglobulins were negatively associated with proteolysis patterns. With well-controlled experimental conditions, this approach offers a label-free strategy to discover signatures of in vivo gut microbial proteolysis uncovering the complex and diverse events of the gut microbiome in health and disease. 